International Journal of Computer Networks \& Communications (IJCNC) Vol.10, No.5, September 2018

\title{
MULTI-CONSTRAINTS ADAPTIVE LINK QUALITY INDEX BASED MOBILE-RPL ROUTING PROTOCOL FOR LOW POWER LOSSY NETWORKS
}

\author{
Sneha $\mathrm{K}^{1}$. and B G Prasad ${ }^{2}$ \\ ${ }^{1}$ Department of Computer Science \& Engineering, BNM Institute of Technology, \\ Bengaluru, Karnataka, India \\ ${ }^{2}$ Department of Computer Science \& Engineering, BMS College of Engineering, \\ Bengaluru, Karnataka, India
}

\begin{abstract}
The importance of IPv6 Routing Protocol for Low power and Lossy Networks (LLNS), also called RPL, has motivated in the development of a robust and quality of service $(Q o S)$ oriented Multi-Constraints Adaptive Link Quality Index (MALQI) based routing protocol. Unlike classical RPL protocols, MALQI enables mobile-RPL while ensuring fault-resilient, reliable and QoS communication over LLNs. MALQI protocol exploits key novelties such as signal strength based mobile node positioning, average received signal strength indicator (ARSSI) and ETX based objective function for fault tolerant best forwarding path selection. The functional architecture of MALQI enables it to be used as the parallel to the link layer RPL that even in the case of link failure can assist efficient data delivery over LLNs. Once detecting link outage, $M A L Q I$ can execute node discover and best forwarding path selection to assist QoS delivery. Contiki-Cooja based simulation reveals that MALQI based mobile-RPL outperforms other state-of-art routing protocols.
\end{abstract}

\section{KEYWORDS}

Mobile-RPL; Adaptive Link Quality; Low Power Lossy Network; Routing Protocol; MALQI.

\section{INTRODUCTION}

The high pace emergence of wireless communication and associated applications has motivated academia- industries to develop more efficient and Quality of Service (QoS) enabling communication solutions. Among major at-hand networking solutions, Wireless Sensor Network (WSN) is found a better candidate because of its decentralized and infrastructure fewer characteristics. WSN is one of the most used communication systems that serve an array of applications including surveillance systems, industrial monitoring, and control, wireless broadcast, defense purposes, traffic surveillance etc. In recent years efforts have been made to introduce mobility with WSN. Introducing mobility in WSN forces network to undergo various adverse situations such as topological changes, congestion, network contention, data drop, retransmission, end-to-end delay, increased latency and energy exhaustion. The situation turns out to be more severe in case of the lossy environment.

In last few years, numerous research efforts have been made to enhance WSN routing protocols [1-5]; however major efforts either intend to deal with energy enhancement, delay sensitive communication or throughput optimization. However, realizing present day application specific demands (i.e., IoT applications and other communication systems operating under LLN conditions) and QoS, the majorities of the traditional approaches either fail to deliver anticipations or provides single performance parameter optimization. To alleviate existing limitations, recently a routing protocol named Routing Protocol for Low Power Lossy network (RPL) was proposed by Internet Engineering Task Force (IETF) in 2008 [6]. RPL is recognized for its efficacy to enhance performance for LLN applications [7]. Typically, LLNs are defined in 
terms of high packet loss, path loss, channel fading, multi-path interferences, low connectivity, low power equipment, etc. Practically, WSN is one of the key examples of LLNs. Considering the significance of RPL a few efforts have been made to use it for LLN applications. Native RPL [6], doesn't have mobility features, and hence to meet the exponentially rising demands mobility aware routing protocol is inevitable. With intend to introduce mobility with RPL a few efforts are made in [8][9]; however, most of the approaches undergo link instability and responsiveness issues due to dynamic topology and network parameters. To ensure the higher performance of a routing model, deciding the best forwarding node (BFN) often plays the vital role. Authors have applied expected number of (control) packet transmission (ETX) as the objective function to perform RPL parent node selection (i.e., resulting the best forwarding path selection). The inefficient BFN selection often results into data drop, delay, and energy exhaustion. On contrary, there are numerous applications where LLN are expected to provide QoS and QoE delivery in a stringent manner while ensuring higher packet delivery ratio (PDR) and low delay. Such expectations might be even at the coarser level or at a fine grain packet level. Applications like threshold crossing alerts, fault or intrusion identification, event detection, burglar alarms, industrial supervision and automation-control, remote healthcare monitoring etc demand higher degree of QoS provision. For example, RPL based healthcare monitoring over LLN signifies a tedious task where there can be mobility of the person whose medical parameters, like electrocardiogram (ECG), pulse rate, temperature etc are being remotely monitored. In such scenarios, the data rates of the order of multiples Kbps may be anticipated to perform health monitoring. IEEE 802.15.4 MAC, a typically applied link layer protocol in RPL-LLNs possesses a built-in ACK mechanism to reduce packet loss during link outage by means of retransmissions. In practice, the highest retransmission count is set-up as 4-5 while retransmission is scheduled at the interval of $10 \mathrm{~ms}$. Though it exhibits retransmission well for the transient link outages for the small duration, it is unable to avoid or stop unsuccessful packet transmission, particularly when the wireless channel has significantly high coherence times or receiver node failures.

Considering the significance of link reliability and dynamic link assessment-based routing, in our previous work, a novel hand-off optimization based best forwarding path selection and routing model was developed. Though, the use of multiple timers and adaptive path selection strengthened our mobile-RPL routing to exhibit higher PDR; however, at the cost of increased control traffic overheads. The increased overhead was due to multiple timers. To alleviate those limitations, in this paper a Multi-constraints Adaptive Link Quality Index (MALQI) model is developed for the best parent node selection and thus the best forwarding path formation over mobility based RPL. MALQI applies ETX as the RPL objective function and additionally uses adaptive RSSI of a node to decide best parent node. In addition, we apply an adaptive RSSI based mobile node positioning measure to strengthen fault-resiliency and reliability. Unlike existing works, where merely default objective function (OF0) are used or expected number of control packet transmission is applied to decide best forwarding path, our proposed MALQI model applies both the adaptive RSSI of the RPL node and ETX that itself is directly related to PDR. Here, the prime motive was to use these two constraints so as to enable reliable best forwarding path formation (i.e., alternative path formation for packet delivery during link-outage conditions). In fact, the proposed MALQI model operates as a parallel functioning unit of link layer where once detecting link-outage it executes node discovery and selects best parent set to form a best forwarding path. In addition, RSSI adaptive mobile node positioning model strengthens our proposed model to ensure fault-resilient communication. Thus, the proposed model can be stated as a link-adaptive routing model where the emphasis is made on enabling link-outage resilient routing decision for higher PDR, low delay, low energy consumption etc. The other sections of the presented paper are divided as follows: Section 2 presents the related works, Section 3 briefs about RPL protocol and key terminologies. It is then followed by problem formulation in Section 4 and proposed model discussion in Section 5. Results obtained are discussed in Section 6. The overall research conclusion and future scopes are discussed in Section 7, and the references used in this manuscript are presented at the end of the paper. 
International Journal of Computer Networks \& Communications (IJCNC) Vol.10, No.5, September 2018

\section{RELATED WORK}

This section briefs some of the key literature presenting RPL routing protocol and associated best forwarding node selection mechanism. Some of the literature discussing mobility based RPL are also discussed. Considering transient link adaptive path selection, Tsiftes et al. [10] observed that a number of data packets are lost during the selection of an alternate parent. Authors stated that with the increase in payloads, hop-counts, mobility and resulting topological variations cause high data loss. Authors [11] applied opportunistic routing protocol for RPL path selection where to enhance reliability and delay they performed transmissions over multiple paths in addition to the native RPL parent-based transmission. A similar effort was made by Duquennoy et al. [12] who exploited an opportunistic routing model for any type of traffic condition. However, authors could not state that how the paths considered by packets fulfill path metric according to the RPL Objective Function (OF).

Considering the significance of mobility with RPL, few efforts have been made [8] [13-17][2023]. Hong et al. in [13] developed a mobility based RPL routing protocol. In their routing model they broadcasted DIO messages containing node rank and link quality index based on which that built DAGs. It enables mobile nodes to identify multiple paths, as an alternative to the failed route. To enable swift local repair, authors used sibling nodes into the route table of the individual RPL node; and in case there is no sibling node available, the node identifying certain broken link can execute global repair. It is performed by the sink, which rebuilds a new DODAG to continue data transmission. To enable swift path formation for data transmission over RPL, Lee et al. [8] derived a transient ETX probing scheme. This approach estimates ETX once finding any new neighbor node and thus builds DIOs and DAOs accordingly (based on the new parent election). This process avoids waiting time for the trickle timer. In addition, they suggested a loop detection and avoidance measure using stamping of the DIO messages with its parent's ID.

Safdar et al. [14] developed mobile a sink-based data collection model, where mobility was controlled in such a way that it moves towards nodes possessing higher energy. It enabled load balancing and avoided network partitioning too. A similar effort was made by Saad et al. [15] who assessed RPL with two different application scenarios, first with mobile sink and second with low-power Power Line Communication (PLC). Authors applied sink movement to avoid retransmission and energy consumption. They made a similar effort in [16] where they applied sink mobility to reduce energy consumption. The key limitation of their model was that it focused only on energy consumption. On the contrary, maintaining higher QoS and QoE is vital for major LLNs applications. In [16], Saad et al. examined sinks mobility in RPL and revealed that sinks mobility can be the potential design criteria; however, it is more effective only for achieving enhanced network lifetime. Authors applied a distributed and weighted moving mechanism for sink mobility in RPL. With intend to reduce the control traffic in RPL network Korbi et al. [17] suggested maintaining lower reactivity to the topological variations. Authors performed mobile node identification and RPL enhancement to achieve better performance. Korbi et al. [17] examined the issue of mobility with RPL over WSN, where to reduce control traffic overhead they performed mobile node identification. It was found vital for efficient path formation and transmission decision. In their other research [17], they derived a dynamic DIS management model for mobility based RPL. In their approach, a condition where a mobile node does not execute new DIO because of rank value also functions by using ETX as the RPL objective function.

Tripathi et al. [18] derived an RPL routing protocol using OMNET++ where they considered ETX as BFN matrix to build the DAG. They found that ETX based route formation can significantly reduce the global repair time and control traffic requirements. Tsiftes et al. [19] examined the performance of the RPL protocol to be used for smart grid applications. Performing 
simulation over OMNET++ they illustrated that trickle timer impacts RPL routing performance, particularly control traffic overhead. Authors stated that the efficiently scheduled trickle timers can reduce latency. Kharrufa et al. [20] introduced mobility with RPL routing protocol for the dynamic network. Authors derived a controlled reverse-trickle timer using RSSI measurements to maintain high network responsiveness and minimum overhead. Based on topological inconsistency, node rebuilds its DODAG to schedules transmission.

Recently, an enhanced RPL routing model called Co-RPL was developed by Gaddour et al. [21] where to incorporate mobility with the RPL, authors exploited the concept of Corona. To examine the efficiency of Co-RPL, authors applied Contiki/Cooja simulation environment. They found CoRPL better than the native RPL. Somaa et al. [22] to derive mobility based RPL proposed an enhanced protocol named Bayesian model Mobility Prediction RPL for LLN application. To achieve it authors performed node identification, node velocity estimation and link duration. This information as cumulative metrics enables efficient forwarding route decision. To provide a reliable RPL routing protocol, Barcelo et al. [23] incorporated Kalman positioning RPL (KPRPL) for WSN. Authors used both static and mobile nodes to form WSN. The prime novelty of KP-RPL model is its efficiency to obtain accurate positioning details for routing decision. Safdar et al. [14] derived a hybrid routing protocol using reactive and proactive mechanism to enhance native RPL with mobile sinks. They found their approach better for handling multiple sink mobility in WSNs. To achieve high PDR, Lohith et al. [24] developed lightweight add-on functionality to strengthen RPL's network layer's data forwarding process. Realizing the impact of trickle timers on control traffic overheads, Lee et al. [25] eliminated the trickle algorithm for DIO and substituted it with the fixed interval ranging from $2 \mathrm{~s}$ to $10 \mathrm{~s}$. These literatures reveal that no doubt a few efforts have been made to enhance mobile-RPL for LLNs; however, the scope for further optimization, particularly for fault-resilient best forwarding path estimation, best parent set selection in the mobile network, etc can't be ignored. This research intends to derive a more computationally efficient and robust mobile-RPL routing protocol for emerging QoS/QoE oriented IoT ecosystems.

\section{RPL: AN OVERVIEW}

Before discussing the implementation of the proposed Multi-Constraints Link Quality Index (MALQI) based mobile-RPL routing protocol, understanding RPL routing is vital. This section briefs about RPL protocol.

Typically, the RPL [6] protocol is stated to be a type of pro-active routing mechanism developed for data transmission over LLNs. RPL operates on the basis of the topological concept of Directed Acyclic Graphs (DAGs). RPL functions by applying three network attributes, DAG Information Solicitation (DIS), DAG Information Object (DIO) and Destination Advertisement Object (DAO) based on which it builds a Destination Oriented Directed Acyclic Graph (DODAG) for data transmission. Here, DAG represents a tree structure that characterizes the default routes in LLNs. The fundamental topological element in RPL is DODAG, which is rooted at a specific node called DODAG root. RPL can have multiple DODAGs that can be characterized in terms of the following parameters:

a) DODAGID: It represents DODAG root.

b) RPLInstanceID: It identifies an autonomous set of DODAGs that can be enhanced for certain specific conditions.

c) DODAGVersionNumber: It increments certain specific events, such as the reconstruction of a DODAG. 
International Journal of Computer Networks \& Communications (IJCNC) Vol.10, No.5, September 2018

d) Rank: It represents the node position with regard to the root node in a DODAG. In DODAG, each node is assigned a rank that typically increases in the downstream direction of the DAG and vice versa.

\subsection{RPL control messages}

The RPL control messages are a type of Internet Control Message Protocol version 6 (ICMPv6) (RFC 2463). There are four types of control messages in RPL. These are:

a) DODAG Information Object (DIO): It is issued by DODAG root to form a new DAG, which is then followed by multicast transmission through DODAG structure. It carries significant network information that permits a node to perform RPL instance discovery, extracting structural parameters, selecting a DODAG parent set, and maintaining the DODAG structure.

b) DODAG Information Solicitation (DIS): It detects neighbour nodes in adjoining DODAGs.

c) Destination Advertisement Object (DAO): It broadcasts reverse-route information (RRIs) to trace the nodes visited along the upward path. Usually, it is transmitted by each node, except the DODAG root to proliferate the routing tables containing prefixes of their children nodes.

d) Destination Advertisement Object Acknowledgment (DAO-ACK): It is transmitted in terms of a unicast packet by a DAO recipient who sends a unicast DAO message as the reply.

The rank information is embedded or routed in DIO in such way that the nodes located farther from the root node would have higher ranks than the near ones. It avoids any looping problem in the network. In DODAG formation RPL applies Objective Function (OF) that decides route selection to achieve targeted anticipation such as high PDR, low, latency and low energy consumption. Typically, OF0 and the expected transmission count (ETX) are used as the OF path metric to estimate node rank with regard to root. As stated above, DIO control message is used to build the upward routes (i.e., from a node to the root). On the other hand, DAO control messages are transmitted from the child nodes to their respective parent node so as to build the downward routes (i.e., from route to the node). DIO message scheduling is performed based on trickle timer [26]. Functionally, trickle timer plays the vital role in RPL such as, it enables swift network convergence while ensuring that the control messages do not over-burden the network. In case of the stable network (i.e., there is no network change) trickle timer increases exponentially so that the re-configuration overheads incurred during topological changes might be reduced. Once detecting any network topological change, trickle timer is reset and network discovery is executed. To join a network, a node requires sending DIS message to advertise its presence in the network.

\section{4 .PROBLEM ForMULATION}

As stated above, enabling best forwarding path selection is vital to ensure reliable and efficient communication over mobile-RPL based LLNs. In mobile-RPL the predominant issue of continuous topological variations and resulting link-outage probability that consequently affects overall network performance. To deal with such adversaries and enable reliable communication with low latency, major existing approaches prefer applying multipath forwarding strategies by performing packet-replication over different links. Though, such an approach could bring about low latency and network convergence, however ensuring link-outage intact remains an open question. In addition, such approaches require significantly high traffic control signals and hence impose computational overheads and energy consumption. Additionally, it can cause 
unpredictable communication among existing RPL DODAG (nodes) that can make network management and routing a complex task. Major existing mobile-RPL routing approaches apply default objective function (OF0) for DODAG formation or forwarding path selection. However, the exceedingly varying network topologies and associated parameter changes, such as signal to noise ratio (i.e., received signal strength index (RSSI)) etc do have impact on link-reliability and performance. To deal with such adversaries, multiple timers and average RSSI based approach can be derived for best forwarding path selection; however, inclusion of multiple timers; connectivity timer, topology aware mobility detection timer, data exchange and hand-off timer, response timer for reliability oriented handoff optimization introduced computational overheads.

As already stated, in mobile-RPL due to dynamic change in topology and network parameters, link-outage become a common threat. To deal with such adversaries, RPL contains two types of link-outage repair model, global and local link repair schemes. The global repair model is executed by the root node where the overall network is re-constructed through a modified DODAG to further re-build the entire network. On the other hand, link level local repair is executed when a parent node undergoes failure. To enable fault-resilient and QoS sensitive transmission, once detecting failure of a parent node an alternate parent can be selected from the parent set table. In case there is no alternate parent node available, node can behave as a new node and can broadcast DIS message. However, link-outage and its consequences leads significant control message overheads, latency, energy consumption, and most significantly low PDR. It significantly put question over the efficacy of the proposed routing model. On the other hand, in case of mobility based RPL undergoes significant changes in topology and hence the link-outage probability can't be ignored. Therefore, to avoid performance degradation, especially reliability of the mobile-RPL network, applying a robust and efficient best forwarding path is of utmost significance. With this motivation, in this research paper we have developed a robust Multi-Constraints Adaptive Link Quality Index (MALQI) based best forwarding path selection model has been developed for mobile-RPL routing protocol.

Realizing the significant limitations or fault-proneness of multipath transmission mechanism over dynamic network topology, it is inevitable to obtain best parent node and the best forwarding path. To achieve it, in this paper a novel link outage resilient MALQI model is developed that functions in parallel to the link layer of the RPL nodes. Once detecting any link outage MALQI is executed that applies ETX as well as adaptive RSSI to estimate best parent node to re-build alternative link so as to ensure reliable data transmission. To achieve it, a backup of the data being transmitted is maintained which is transmitted through alternate best forwarding path (with the help of best parent node) towards destination. Realizing the resource constrained communication, once receiving acknowledgment from the sink, the buffer storing backup data can be flush to accommodate packet during future outage condition. Here, it should be noted that MALQI can operate in parallel to the network layer and the parent nodes in the table can be ordered based on path metrics (i.e., ETX and Adaptive RSSI). In proposed model, the alternate parent selection process (to build best forwarding path) continues iterating based on available resource constraints and QoS demands. One of the key parameter that affects the overall performance is the MAC retransmission limit that decides when to execute node discovery for best parent node selection and best forwarding path formation. In addition, the estimation of adaptive RSSI and its use for PDR estimation, which is later used to decide ETX makes proposed model robust enough to ensure link-outage resilient and reliable communication. Here, higher RSSI signifies higher PDR and hence QoS assurance. Thus, consideration of PDR for ETX decision makes our proposed routing model strong enough to build link-outage resilient path for communication using mobile-RPL over LLNs. In addition, for a pre-defined timeout delay between retransmissions, a relatively lesser retransmission limit can compel node to impulsively run off from the current parent to identify a parent with relatively low link quality. On the contrary, higher retransmission limit can force node to continue with the parent undergoing link 
outage even for the long time. In our research model parent node are selected as per native RPL model where it contains the parent nodes allied with each DODAG. Here, parent nodes are ordered in decreasing order of their respective path metrics, estimated by means of ETX objective function. In addition to the above-mentioned approaches, the implementation of RSSI adaptive mobile node positioning can be of paramount significance to enable timely (i.e., delay resilient) data gathering and reliable transmission over LLNs.

\section{PROPOSED MODEL}

This section briefs our proposed Multi-Constraints Adaptive Link Quality Index (MALQI) based link-outage resilient best parent node selection and the best forwarding path formation approach for mobile-RPL to be used in LLNs. As stated in previous sections, once detecting any linkoutage or failure in delivering the packet to the parent node, MALQI which operates in parallel to the network layer enables forwarding the same data packet to the defined parent node. It is achieved by identifying best forwarding path with the help of parent node (in the same DODAG) ordered with respective path metrics. In our proposed approach, the node discovery is initiated only after getting link-outage adversary. The best parent node selection-oriented node discovery continues iterating to identify best forwarding node based on respective resource constraints and QoS sensitive demands (i.e., higher PDR and link reliability). One of the contributions is that the proposed MALQI routing model maintains backward-compatibility with the native RPL routing scheme. Unlike major existing approaches where either ETX or OF0 is used as RPL objective function for best parent node selection, in this paper multiple constraints including ETX and adaptive RSSI of a node are considered for the best parent node selection. Since higher RSSI signifies higher PDR that itself is related to the ETX count and hence dynamic measurement of RSSI of a node and applying it to derive ETX for best path selection makes our proposed model robust to avoid any link outage probability. As already stated MALQI operates in parallel to the network layer of the RPL node and hence once detecting any link outage it is executed that further initiates node discovery to identify best parent node from parent set table. In our proposed model, based on respective path metrics a list of parent nodes are stored pro-actively in parent table. The parent table maintains the parent nodes related to each DODAG, where each parent node is ordered in the decreasing order of its path metrics (i.e., ETX and Adaptive RSSI). It enables the swift path decision to achieve better performance. In our proposed model, in case MALQI doesn't find any parent node in parent set table it executes node discovery, which is then followed by the formation of the best forwarding path (as the alternate path for data transmission).

Considering the overall operating model for the proposed MALQI based IP layer packet forwarding path is depicted in Fig. 1. In this figure IP layer, its interface with the link layer and RPL can be visualized precisely 


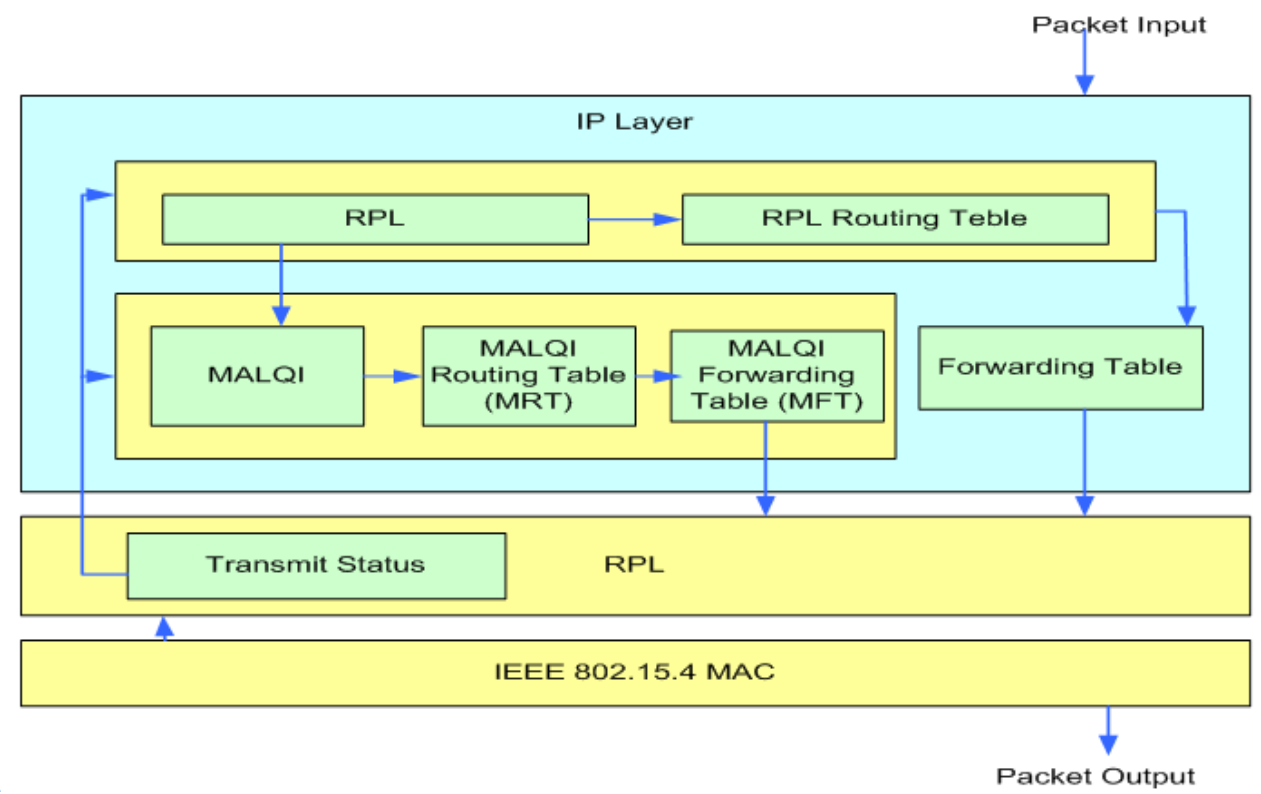

Fig. 1. MALQI based packet forwarding in mobile-RPL

Realizing the significance of node positioning and respective routing decision, in this paper, we have derived RSSI based mobile node localization. Since MALQI exploits the adaptive RSSI value of each RPL node and hence using it for node positioning is not an intricate task. The discussion of the RSSI based node localization is presented in the following sub-sections. Meanwhile, since the proposed LLN environment comprises both the static nodes (i.e., access points or the anchor nodes) as well as the mobile node, and hence discussing inter-node communication is must. The following sub-section briefs about the node positioning and internode communication in the proposed routing protocol.

\subsection{System Model}

For any communication system node deployment and its optimal localization play a vital role in ensuring higher performance. Unlike random node deployment we have incorporated a confidence region-based node positioning; however, the proposed MALQI based mobile-RPL protocol can function with any network topology. A brief of the applied LLNs node positioning model is presented as follows:

\subsubsection{Node Positioning}

In targeted LLNs the static nodes, usually called anchor nodes are placed in such way that the node location doesn't contain error. This, as a result, can enable swift network parameter estimation, decision process and forwarding path selection. Considering mobility across the network, estimating the location of the mobile node is vital to ensure optimal performance and QoS delivery over LLNs. For example, with an application specific scenario when a mobile sink intends to collect data for further processing or decision process, it requires collecting data timely and reliably. In such circumstances the dynamic positioning of the mobile node (in conjunction with best forwarding node or parent node selection) is the must. To achieve it, the mobile node can consider RSSI of an anchor node as the parameter to decide whether to move towards that for link formation or not. In our proposed localization model, the mobile node positioning is performed by considering the adaptive or dynamic RSSI value of an anchor node. This section briefs about the localization of nodes across the network and respective routing decision (routing decision for inter-static or inter-anchor routing decision and mobile to anchor routing decision). 


\subsubsection{Confidence Region (CR) based Node Localization}

This section primarily discusses the mobile node positioning under a mobility condition. In our research, we use dynamic RSSI of an anchor node to perform mobile node positioning. The prime reason of the selection of this method is that unlike other approaches (for example, least square), it estimates and characterizes the impact of each static or anchor node distinctly. In addition, the RSSI estimates from nearer static nodes influences node positioning significantly in comparison to those estimated from distant static nodes. To create CR, we consider that the mobile nodes are aware of the time-varying channel statistics follows the long-distance path loss model. Here, RSSI at certain instant (say, $K^{\text {th }}$ time slot) is obtained as equation (1).

$$
\text { RSSI }^{k}=P_{1 m}-10 \alpha \log _{10} d-\gamma
$$

Where $\mathrm{P}_{1 \mathrm{~m}}$ refers the received signal at a pre-defined distance (here, 1 meter from the source), $\alpha$ refers the path-loss exponent, the transmission range is d while, $\gamma^{\sim \mathcal{N}}\left(0, \sigma_{\gamma}^{2}\right)$ signifies zero mean Gaussian noise. The derived expression for the distance between the mobile and the static node using RSSI is given in equation (2).

$$
\hat{d}_{R S S I}^{k}=10^{\frac{R S S l^{k}-\mathrm{p}_{1 \mathrm{~m}}}{10 \alpha}}
$$

Here, it must be noted that $\hat{\mathrm{d}}_{\mathrm{RSSI}}^{\mathrm{k}}$ avoids following a Gaussian distribution because of the exponential relationship between RSSI and distance. Practically, the distance estimation model applies a log-normal distribution. Mathematically it is given in equation (3),

$$
\ln \hat{d}_{R S S I}^{k} \sim \mathcal{N}\left(\ln d^{k}, \sigma_{d}^{2}\right)
$$

Where $\mathrm{d}^{\mathrm{k}}$ refers the distance to static node and the standard deviation of the distance estimated ${ }^{\sigma_{\mathrm{d}}}=\left(\sigma_{\mathrm{\gamma}} \ln 10\right)=(10 \alpha)$. Thus, the probability density function (PDF) is estimated using equation (4).

$$
f d(\hat{d})=\left\{\begin{array}{l}
\frac{1}{\sqrt{2 \pi} \sigma_{d} \hat{d}} \exp -\frac{(\ln \hat{d}-\ln d)^{2}}{2 \sigma_{d}^{2}} \text { if } \hat{d}>0 \\
0 \quad \text { if } \hat{d} \leq 0
\end{array}\right.
$$

Where $\mathrm{d}$ refers the real estimated distance. Thus, retrieving PDF of the distance measure, the CR region of the a-th static node is estimated. Here, we represent $C R$ as $C_{a}$ that signifies the area in which the mobile node is present with confidence likelihood $\mathrm{q}_{\mathrm{LN}}$. Now, considering omnidirectional antennas, CR presents a circle with radius $\mathrm{r}$ centered over a-th static node position, while fulfilling the following condition

$$
\operatorname{Pr}\left\{d^{k} \leq r\right\}=q L N
$$

We derive $\mathrm{q}^{\mathrm{LN}}$ as equation (5)

$$
q L N(\mathrm{r}) \simeq \int_{0+}^{\mathrm{r}} \frac{1}{\sqrt{2 \pi} \sigma_{d} x} \exp \left(-\frac{\left(\ln x-\ln \hat{d}_{R S S l}^{k}\right)^{2}}{2 \sigma_{d}^{2}}\right) \mathrm{d} x
$$

Thus, the m-th mobile node characterizes its CR by overlapping CRs of the L nearest static nodes as shown in equation (6). Mathematically, 


$$
C_{m}=C_{1} \cap C_{2} \cap \ldots \ldots \cap \cap C_{2}
$$

In our node positioning model, mobile node estimates its location, $\widehat{\mathrm{Pm}}$ within its $\mathrm{CR}$ by estimating the discrete points distributed uniformity within $\mathbf{C}_{\mathrm{m}}$. The mobile node calculates the probability to be present in each point of $\mathrm{C}_{\mathrm{m}}$ using PDF, as estimated by (4). Assuming that the PDFs of different static nodes are autonomous, the mobile node estimates its location using equation (7).

$\hat{\mathrm{P}} m=\max _{c} \prod_{a \in A^{p}} q L N\left(d_{c, a}\right) \forall c \in C_{m}$

Where $\mathrm{A}^{\mathrm{v}}$ represents the set of static nodes used for mobile-node positioning. The other variable $d_{c, a}$ presents the Euclidean distance between c-th point to a-th static or the anchor node.

\subsection{MALQI mobile-RPL}

Our proposed MALQI based mobile-RPL protocol performs routing in following two phases:

a) Inter-Static Node Routing (ISNR)

b) Mobile-to-Static Node Routing (MSNR)

A brief discussion of the applied routing mechanisms is given as follows:

\subsubsection{Inter Static Node Routing}

In this paper we have applied MALQI based RPL routing model to perform data transmission between anchor nodes or the static nodes. However, since inter-anchor communication doesn't involve node mobility and hence link outage probability is very confined. It states that even native RPL can be efficient to perform communication between static or anchor nodes. In our research work, the DODAG is formed using DIOs control messages. Being MALQI based routing; we estimate the node rank by measuring both ETX as well as current RSSI. Thus, these multiple constraints are used as the objective function to identify or select best parent node, which is then followed by the selection of best forwarding path for data forwarding or transmission. Here, a node $\mathrm{i}$ at first measures RSSI of the node in parent set based on which the PDR is estimated. Finally, using estimated PDR the ETX objective function is calculated using (8). The total end-to-end ETX counts from i source or transmitter or the jth forwarding node is obtained as $\mathrm{ETX}_{\mathrm{ij}}$ in equation (8).

Mathematically,

$$
E T X_{i}=\frac{1}{P D R_{i, j}}+E T X_{j}
$$

Where, ETX $_{\mathrm{i}}$ represents the total ${ }^{\text {ETX }}$ required to reach jth static node. The other variable ETX $_{j}$ refers the total ETX of the jth node. Here, the ETX between the two nodes, ${ }_{i}$ and $j$ is estimated as the reciprocal of PDR. i.e. $\operatorname{ETX}_{\mathrm{ij}}=\frac{1}{\mathrm{PDR}_{\mathrm{i}, \mathrm{i}}}$.In addition, when making best forwarding path selection decision, the anchor or static node estimates current RSSI of the neighboring node. The 
consideration of ETX as well as current RSSI for best parent node selection and forwarding path selection strengthen routing protocol to assure reliable and QoS communication over LLNs.

\subsubsection{Mobile to Static Node Routing (MSNR)}

In fact, the native RPL is designed with static node only and therefore it does not possess strategic measures to deal with mobility in network topology. In addition to the energy exhaustion, computational overheads and delay, control signaling required for maintaining or assuring QoS communication becomes too complex with mobility based RPL [27]. To alleviate this issue, in this work we have developed a multi-constraints adaptive link quality index (MALQI) based mobile-RPL routing protocol. Additionally, the inclusion of positioning information enables our proposed routing model robust enough to ensure reliable communication over mobile-RPL. The mobile nodes select their parent node based on MALQI model that estimates RSSI based ETX count and current RSSI of the node to decide parent node. In our model, when transmitting packet from ath static node, the total end-to-end ETX for mth mobile node (say,ETX $\mathrm{m}_{\mathrm{m}}$ ) is estimated as the sum of ETX from node itself to the ath static node $\left(\mathrm{ETX}_{\mathrm{m}, \mathrm{a}}\right)$ and the ETX from static to the destination $\left(\mathrm{ETX}_{\mathrm{a}}\right)$ shown in equation $(9)$ :

$$
E T X_{m}=E_{T, a}+E T X_{a}
$$

Additionally, ETX $_{a}$ can be retrieved from the rank of the a-th static node estimated using (8). Noticeably, in the proposed routing model the key parameters such as the rank information, ETX, current RSSI are embed in the field of DIO control packet. We have estimated ETX $\mathrm{m}_{\mathrm{m}, \mathrm{a}}$ as the reciprocal of PDR m,a $_{\text {. }}$ PDR is measured as the likelihood of getting a packet successfully at kth time slot with RSSI more than the transceiver's sensitivity $\left(\mathrm{RSSI}_{\mathrm{th}}\right)$ [28]. Mathematically shown in equation (10):

$$
P D R_{m, a}=\operatorname{Pr}\left\{R S S I_{m, a}^{k} \geq R S S I_{t h}\right\} .
$$

Assuming decaying RSSI over distance (log-distance path loss model), PDR $_{\mathrm{m}, \mathrm{a}}$ is obtained as equation (11).

$$
P D R_{m, a}=\operatorname{Pr}\left\{\gamma \leq P_{1 m}-10 \log _{10} d_{m, a}-R S S I_{t h}\right\} .
$$

As, $\gamma$ refers Gaussian distribution $N\left(0, \sigma_{\gamma}^{2}\right), \mathrm{PDR}_{\mathrm{m}, \mathrm{a}}$ is estimated using equation (12).

$$
P D R_{m, a}=\operatorname{Pr}\{\gamma \leq X\}=\int_{-\infty}^{X} \frac{1}{\sqrt{2 \pi} \sigma_{\gamma}} \exp \left(-\frac{\gamma^{2}}{2 \sigma_{\gamma}^{2}}\right) d \gamma,
$$

In our model $X$ is used to enable clarity rather using $P_{1 m}-10 \alpha \log _{10} \hat{d}_{m, a}-R S I_{\text {th }}$.

In our model, $\mathrm{d}_{\mathrm{m}, \mathrm{a}}$ is replaced by $\widehat{\mathrm{d}}_{\mathrm{m}, \mathrm{a}}$, Thus, exploiting RSSI information and associated PDR value, mth mobile node selects its parent node ${ }^{\mathrm{a}_{\mathrm{m}}}$ using following mathematical formulation shown in equation (13):

$$
\mathrm{a}_{\mathrm{m}}=\arg \min _{\mathrm{k}}\left(\frac{1}{\operatorname{PDR}_{\mathrm{m}, \mathrm{k}}}+\operatorname{ETX}_{\mathrm{k}}\right), \quad \forall \mathrm{k} \in \mathcal{K}_{m}
$$


The detailed discussion of the MALQI routing approach and associated functionalities is presented in the following sub-sections.

\subsection{MALQI mobile-RPL Based Routing Mechanism}

Generally, in case of the static network type, the RPL estimates the ETX value for each node and measures the link quality to decide best parent node. Applying estimated link quality or link information about its neighboring node, it identifies the parent node by extracting DIO messages, where DIO message contains significant ETX, RSSI and rank information. In addition, based on application-specific scenario, there can be only source node as the mobile over certain statically established relay network or all nodes as in mobile vehicular communication. In such cases, RPL node might not have the precise information about the link quality of its parents. This is because the parent nodes itself might be varying temporally and spatially. It enables the selection of the parent node as highly difficult. Some of the key reasons are the reduced DIO frequency that confines node from discovering the availability of the new parent node (i.e., parent set). Even if it exhibits it, as the node has not sent the packets to any of the parents from the updated parent-list or parent-set, there is no significance of estimating the link ETX of these parents for the best parent selection. Considering these intricacies over real-world network scenario, with mobileRPL our proposed routing model incorporates ETX and current RSSI as path metrics for best parent node selection. Here, the rationale of using this multiple constraint is twofold. Since RSSI characterizes the present link quality, which is significantly related to the PDR to be used for ETX estimation [29], its consideration is vital to ensure reliable and optimal packet transmission through best alternate parent (and thus best forwarding path). Since, the estimation of RSSI can be done directly from the incoming DIO messages transmitted from the neighboring nodes that additionally comprises rank information can play vital role in estimating path metric. However, depending on DIO messages for the current link information might result into disabling the trickle timer [17]. Therefore, in this paper our proposed MALQI based mobile-RPL routing protocol the trickle timer is disabled and the DIO messages are transmitted periodically. This section briefs about the algorithmic and implementation aspects of the proposed routing approach named MALQI.

To execute MALQI over mobile-RPL, our proposed routing model ensures that each node in a DODAG maintains the list of its parent node based on respective path metrics. Additionally, we consider that the protocol stack facilitates required connectivity at the network layer to retrieve information about the link layer transmission. Once detecting any link outage due to mobility, the link layer of the RPL node executes MALQI in parallel (that functions in parallel to the link layer). Our proposed MALQI model at first examines the parent nodes in proactively updated parent set where parent nodes are updated in the decreasing order of respective rank and RSSI value. Here, finding link outage RPL nodes assess each parent node for its suitability for being parent node, and thus identifying best parent node it rebuilds best forwarding path to transmit data packets. Noticeably, the newly formed path functions as alternative to the path underwent link outage (due to mobility or resulting adversaries). In case a RPL node does not find optimal best parent node, it behaves as a new node and thus MALQI executes node discovery phase. In node discovery sending multicast beacon message it retrieves path metrics and thus applying multiple contestants as RPL objective functions (i.e., MALQI) it performs best parent node. Once obtaining the best parent node, a parallel best forwarding path is constructed through which the data communication is made. Thus, the overall mechanism ensures link-outage resilient or fault resilient routing model for mobile-RPL to be used in mobility based LLN applications. The following figure (Fig. 2) presents the proposed MALQI model based best forwarding path selection and data transmission over mobile-RPL. 


\section{AIGORITHM:}

Packet $_{\text {current }}$ : Data Packet to be transmitted

Parent $_{\text {current }}$ : Receiving node of the Current data packet.

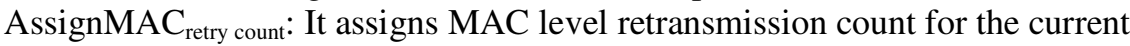
packet Packet current $_{\text {for Parent }}$ current $_{\text {f }}$

Select alternate parent Parent $\mathrm{alternate}_{(}()$for BFN based transmission

Retrieve best Parents $\max _{\operatorname{ma}}$

for each packet do

Parent $_{\text {current }}=$ Set as default preferred parent

Parents $_{\max }=$ Retrieve Parents $_{\max }($ Step-5)

Parent ${ }_{\text {attempted }}=0$

Obtain data backup for Packet current $_{\text {}}$

Initiate Transmission $\mathrm{T}_{\mathrm{x}}$;

Assign MAC $_{\text {retry count }}\left(\right.$ Packet $_{\text {current }}$, Packet $\left._{\text {current }}\right)$

Transmits (Packet current $_{\text {) }}$

Par attempted++

Wait for ACK (MAC T xstatus()) $_{\text {( }}$

if MAC $\mathrm{T}_{\mathrm{xstatus}=1}$ then

Release backup data or free buffer ${ }_{\text {backup }(}$

else

Update Parent current $_{\text {t }} \leftarrow$ Parent $_{\text {alternate }}$

if $\left((\right.$ Parent current $) \|\left(\right.$ Parent $_{\text {attempted }}>$ Parents $\left.\left._{\max }\right)\right)$

then

Release backup data or free buffer ${ }_{\text {backup }(}$

end if

Initiate transmission $\mathrm{T}_{\mathrm{x}}$

end if

end for

Fig. 2. MALQI based data best forwarding path selection and data transmission

In summary, the overall proposed system can be summarized as: once network layer receives a packet to transmit, the native RPL executes first, and once finding link-outage at certain time step or instant MALQI-RPL comes into existence and estimates the parent and best forwarding path for forwarding the packet. Being a mobile node based RPL, the sensibility of the proposed routing model is affected significantly, and its efficacy is characterized by how swiftly it can react to the channel conditions in case of any link-outage condition. In our proposed model, since for each new data packet is being transmitted to the selected RPL parent, we follow the RPL generated path under normal channel conditions. Once detecting any link-outage or parent failure MALQI executes node discovery and identifies the alternate parent. In our proposed routing model, MALQI is executed when a node fails to forward its data to the current parent within the predefined maximum MAC-level retransmission limit.

\section{RESUlTS AND DISCUSSIONS}

Considering the significance of mobility based RPL routing protocol for Low Power Lossy Networks (LLNs) applications, in this paper we developed an efficient and robust routing model that considers almost every inevitable functional behavior of the routing approach. In this research model, the predominant emphasis was made towards developing multiple constraints based best parent selection and associated best forwarding path formation, link quality adaptive node positioning etc. These all novelties enabled our proposed Multi-constraints Adaptive Link 
Quality Index (MALQI) routing model to exhibit reliable data transmission over mobile-RPL, even under link-outage conditions. Realizing the direct impact of RSSI on PDR and associated ETX count, in our model RSSI adaptive ETX was considered as decision variable for best parent node. In addition, to enable reliable link connectivity (so as to avoid any link outage due to mobility caused dynamic topology) dynamic RSSI of each neighbouring node is selected as the second constraint for parent node selection. Based on adaptive RSSI value, a parent set table is formed that stores parent nodes in decreasing order of RSSI level. Once the node (link layer) detects the link-outage it executes network discovery and retrieves the best parent node from the parent set node table. In this way, identifying best parent node and forming best forwarding path the node transfers its data to the destination. Here, it should be noted that the prime objective is made on enabling reliable data communication over mobile-RPL, and therefore node maintains a backup of data to be transmitted, and hence applying alternate best forwarding path it transfers the data to the destination. One more calibrated approach applied in our model was the avoidance of varying Trickle timer that avoids significant control message overheads and resource consumption. Here, we have considered trickle timer at a fixed rate (i.e., $2 \mathrm{~ms}$ ).

The overall proposed MALQI enriched mobile-RPL protocol is developed using Contiki-Cooja Simulator, where it (MALQI) functions as a network plug-in. In fact, our proposed MLAQI model functions as a plug-in over the RPL network protocol stack of Contiki. The overall simulation model was developed and examined over Contiki-2.7 platform. One noticeable feature of the proposed model is its lightweight nature of the implementation, in which it takes only $2 \mathrm{~KB}$ of flash memory to implement MALQI over entire resource constrained RPL motes (i.e., Sky mote). To assess the efficiency of the proposed mobile-RPL routing approach, the overall performance has been examined in terms of packet delivery ratio (PDR), delay, node density versus PDR, and payload versus PDR, etc. To perform graphical presentation of the results, we have used MATLAB 2015 a platform, where the consolidated outputs for different parameters are given as input, and accordingly performance evaluation is done. To examine the performance of the proposed mobile RPL routing protocol and best forwarding path selection model, two test cases have been considered. In the first case the performance of the proposed MALQI-RPL has been compared with the native RPL. On the other hand, in the second RPL protocol MALQ-RPL performance is compared with other state-of-art mobile-RPL routing protocol.

\subsection{MALQI-RPL Vs Native RPL}

In this test case, our proposed Multiple Constraints Adaptive Link Quality Index (MALQI) based mobile-RPL routing protocol, as well as the native RPL routing protocol, were examined for respective efficiency for mobile routing purpose over LLNs. MALQI considers ETX as well as adaptive (i.e., current) RSSI of a node to decide best parent node for forwarding path selection. On the contrary, native RPL considers ETX as the sole objective function for parent selection. Fig. 3 presents the performance of the proposed routing protocol in terms of packet delivery ratio (PDR) when the varying number of packet transmission or payload. Looking into the results (Fig. 3 ), it can be found that the proposed MALQI-RPL routing model exhibits almost or near $100 \%$ throughput, on the contrary, native RPL under mobility exhibits relatively lower PDR. Here the prime reason of reduced PDR can be the parent node selection issue. No doubt, with generic or native RPL there could be delay in identifying best parent node and forwarding path, this as a result could lead data drop. Proposed MALQI routing model initiates node discovery once identifying the fault or link outage, in that case since the proposed routing model encompasses parent set table and initially identifies best RPL node for alternate path formation. Due to this robustness, MALQI avoids transient node discovery and hence achieves low latency or delay (Fig. 8). Consequently, it helps in forming an alternate forwarding path swiftly and hence provides higher throughput. In major communication scenario, especially for real-time applications there used to be varying payload, and hence adaptive a best forwarding path to carry 
the at-hand packets or load is the must. We have examined the performance of MALQI-RPL routing protocol by varying packets per second. Here, we have examined relative PDR by varying packet rate (i.e., 1 packet/sec, 2 packets/sec, 4 packets $/ \mathrm{sec}, 8$ packets $/ \mathrm{sec}$ and 10 packets $/ \mathrm{sec}$ ). Observing Fig. 4, it can be visualized that the proposed MALQI-RPL based routing model exhibits higher PRR than the native RPL. The result depicts that the proposed mobile-RPL routing model is capable of sustaining higher PRR even under increasing packet rate. Here, not only the efficacy of multiple constraints and adaptive RSSI (say, current link quality) based best parent selection and path formation technique strengthen the proposed routing model, but also the link quality adaptive mobile node positioning enables optimal node movement. This consequently enables efficient data gathering and the consideration of adaptive link enables reliable communication to assure low packet loss and hence higher PRR.

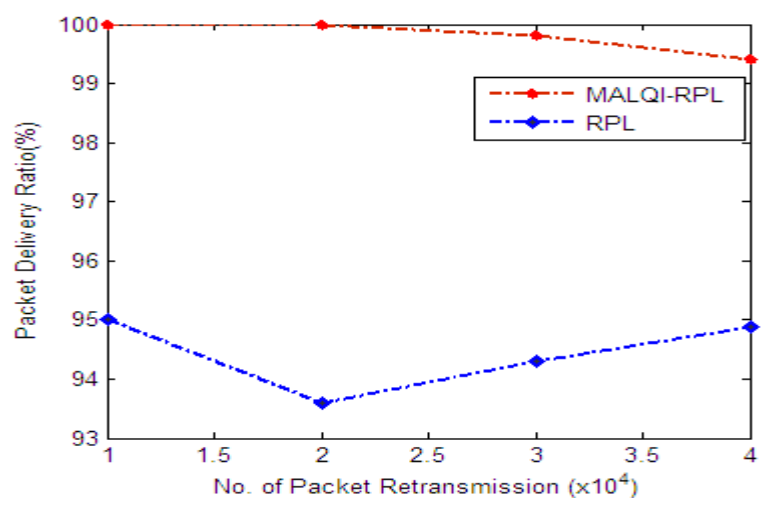

Fig. 3. Packet Delivery ratio Vs number of packet retransmission

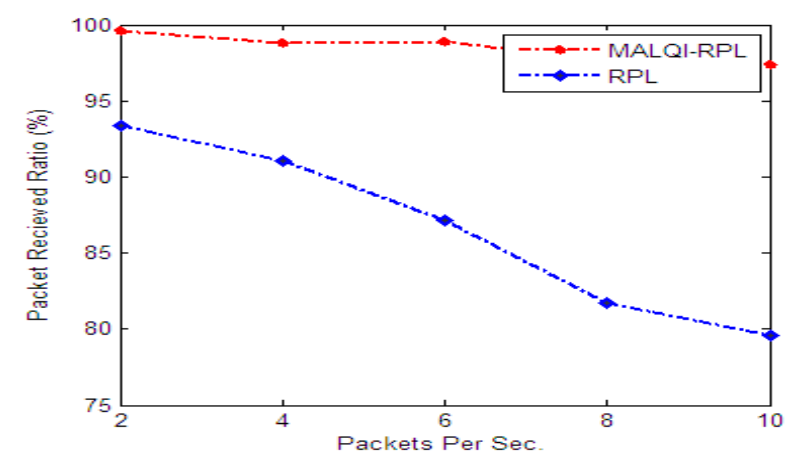

Fig. 4. Packet Received Ratio (PRR) Vs packets per second

Fig. 5 presents PDR performance while varying packet injection rate or number of packets variation. Due to mobility in LLNs there can be dynamic network parameter variations, topological changes and link variation causing link outage and data loss. Since, in our research we emphasized on assuring minimum data loss even under mobility or link-outage probability, our proposed MALQI-RPL routing protocol has accomplished anticipated performance. Fig. 6 confirms that the proposed routing model is capable of delivering optimal packets to the destination while ensuring minimal packet loss, even under varying payload or packet injection rate. This is because of the link adaptive parent node selection and swift best forwarding path selection. 


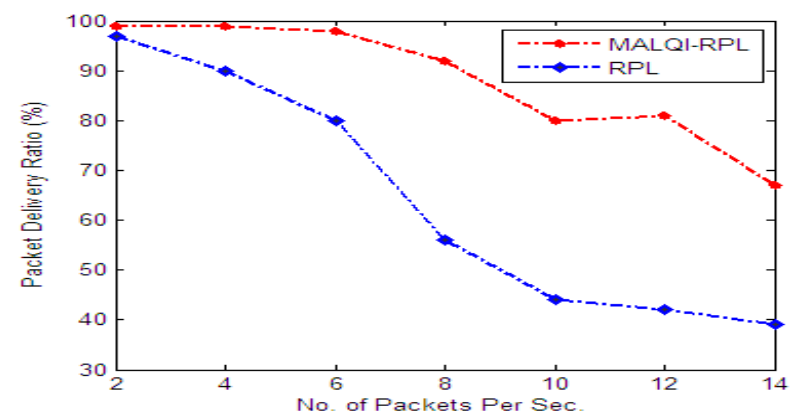

Fig. 5. Packet delivery ratio Vs packet injection rate $(\mathrm{p} / \mathrm{sec})$

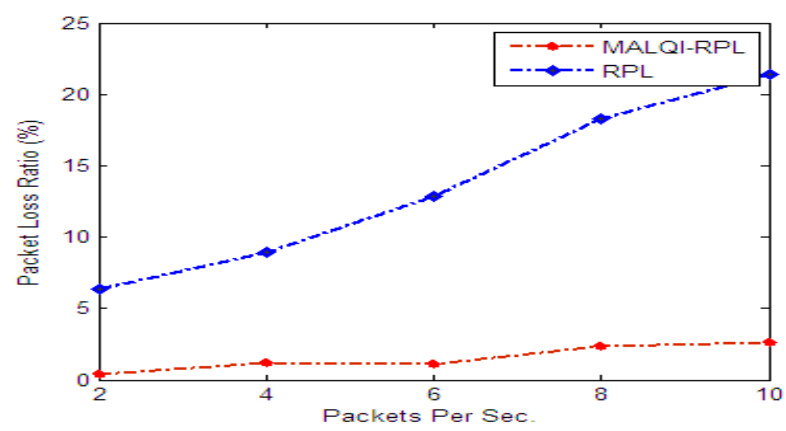

Fig. 6. Packet loss rate Vs packets per second

No doubt, maintaining minimum number of hops often gives confidence to have higher performance and reliability. Low hop counts not only reduces the probability of link-outage due to mobility but also alleviates the likelihood of data loss. To achieve higher PDR or reliable data transmission our proposed MALQI-RPL routing model has exhibited lower loss than the native RPL based routing (Fig. 7). The native RPL routing protocol with higher hops or ETX undergoes link outage due to mobility, and hence suffers higher packet loss (Fig. 7). On contrary, the proactive parent node table management and MALQI-RPL based best parent node selection strengthen proposed routing model to assure low packet loss. Additionally, the implementation of link quality (i.e., current RSSI) adaptive mobile positioning also ensures alleviation of the link outage. This as a cumulative outcome assures minimum packet loss, as depicted in Fig. 7.

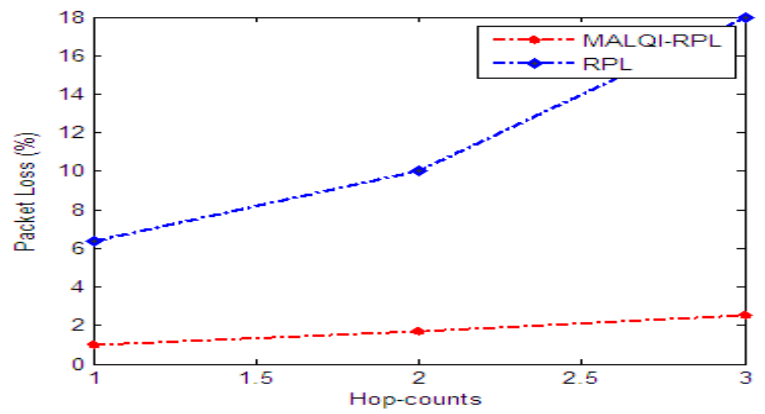

Fig. 7. Packet loss Vs Number of Hops

For any QoS or QoE centric communication requirement, maintaining minimum latency or endto-end delay is must. The simulation result as presented in Fig. 8 confirms that the proposed MALQI-RPL based routing protocol performs better time efficient best route formation and data transmission. Here, the delay efficiency is because of enhanced swift best parent node selection 
and forwarding path selection, even under mobile scenario. Thus, observing overall results for the proposed MALQI-RPL routing protocol and the state of art native-RPL routing protocol; it is evident that the proposed MALQI-RPL approach is robust, reliable and time efficient for LLNs under mobile conditions.

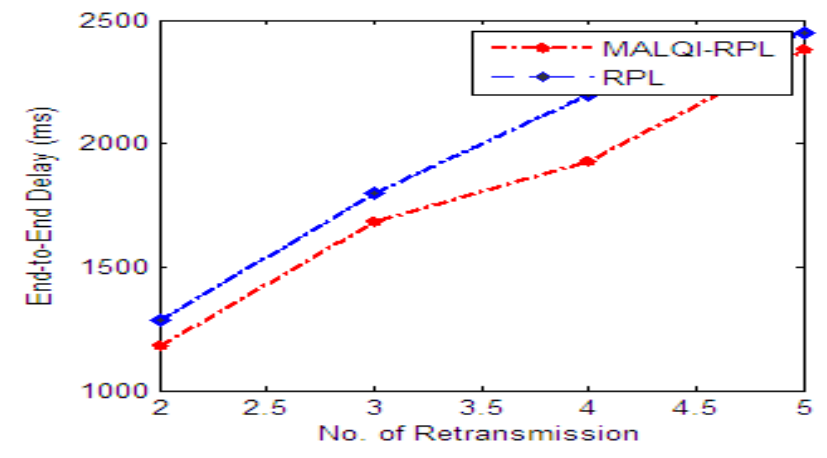

Fig. 8. End-to-end delay Vs number of retransmission

In the above discussion our proposed MALQI-RPL routing protocol was evaluated and assessed for relative performance. In above results, the proposed routing model has exhibited better than the state-of-art native RPL based routing. However, to further explore the effectiveness of the proposed routing approach (i.e., MALQI-RPL), we have compared the results with an existing mobile RPL routing protocol called Co-RPL. The comparative performance is discussed as follows:

\subsection{MALQI-RPL Vs Co-RPL}

We have compared the performance of our proposed MALQI-RPL routing protocol and the existing mobile-RPL protocol called Co-RPL (Co-RPL: RPL Routing for Mobile Low Power Wireless Sensor Networks using Corona Mechanism [21]). To enable mobility over LLNs using RPL, Co-RPL protocol [21] performs mobile node tracking and its position estimation. In fact, authors [21] applied the corona mechanism to enable RPL router localization under mobility scenario. However, they could not consider varying link quality, adaptive RSSI based best parent selection, and more importantly, authors lacked exploiting the significance of RSSI adaptive mobile node localization or positioning. Unlike Co-RPL, in our proposed multi-constraints adaptive link quality index (MALQI) RPL protocol, we have applied link quality based mobile node positioning, multiple constraints (i.e., ETX and current RSSI of the RPL node) based best parent node selection and DAG formation so as to enable optimal best forwarding path formation. In this way, hypothetically, our proposed model must exhibit more reliable and efficient communication than the existing Co-RPL routing approach. To examine this, we have compared the average performance of the proposed MALQI-RPL and Co-RPL routing protocols. The results obtained are given as follows:

Fig. 9 presents the PDR performance comparison between the proposed MALQI-RPL routing protocol and Co-RPL protocol for mobility based LLNs. As depicted in Fig. 9, it can be visualized that the proposed MALQI-RPL protocol exhibits more PDR than the Co-RPL. This can be due to the multiple constraints, particularly adaptive link quality-based parent node selection and forwarding path selection. Additionally, the impact of adaptive RSSI based mobile node positioning can't be ignored to have influence of enhanced PDR over the existing system. 
International Journal of Computer Networks \& Communications (IJCNC) Vol.10, No.5, September 2018

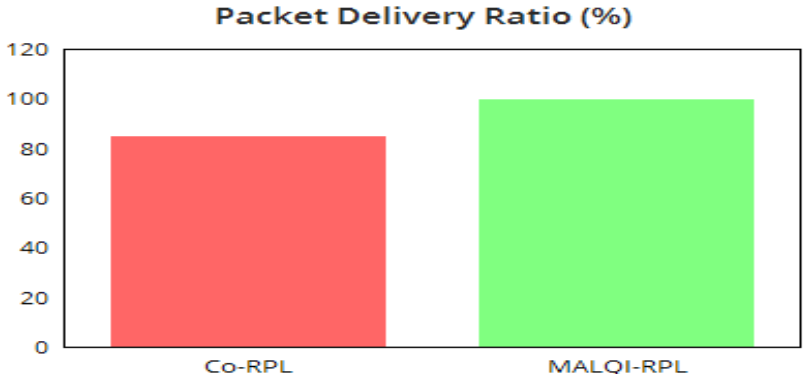

Fig. 9. PDR performance comparison between MALQI-RPL and Co-RPL

To examine the effectiveness of the proposed MALQI-RPL routing protocol for varying network traffic rate, we have assessed the packet loss rate variation with changing load. The average packet loss ratio obtained reveal that our proposed routing protocol performs better than Co-RPL. Fig. 10, Fig. 11, and Fig. 12 present the average packet loss observed using our proposed MALQI-RPL and co-RPL routing protocols for the date rate of $1 \mathrm{p} / \mathrm{s}, 2 \mathrm{p} / \mathrm{s}$ and $3 \mathrm{p} / \mathrm{s}$ respectively. The overall average packet loss is presented in Fig. 13, where it is revealed that the proposed routing protocol outperforms state-of-art technique Co-RPL.

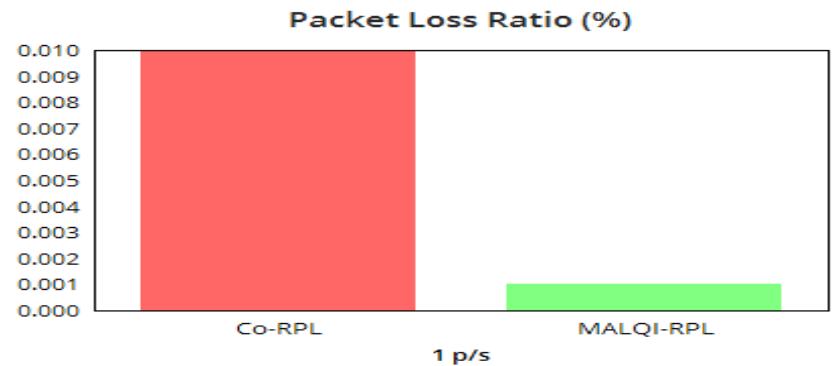

Fig. 10. Packet Loss ratio comparison between MALQI-RPL and Co-RPL with $1 \mathrm{p} / \mathrm{s}$ data rate

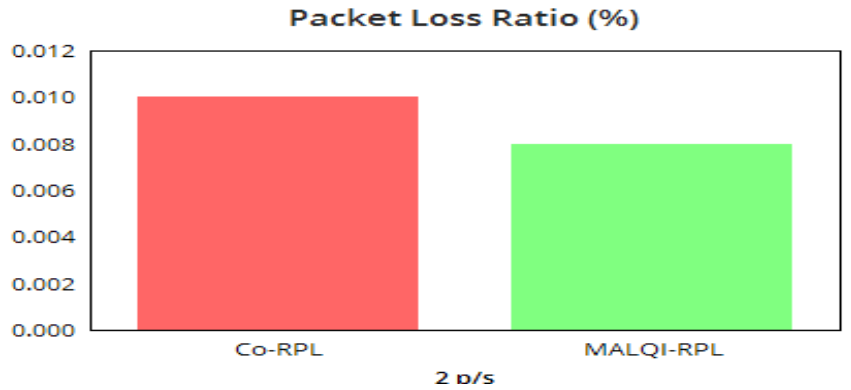

Fig. 11. Packet Loss ratio comparison between MALQI-RPL and Co-RPL with $2 \mathrm{p} / \mathrm{s}$ data rate 
International Journal of Computer Networks \& Communications (IJCNC) Vol.10, No.5, September 2018

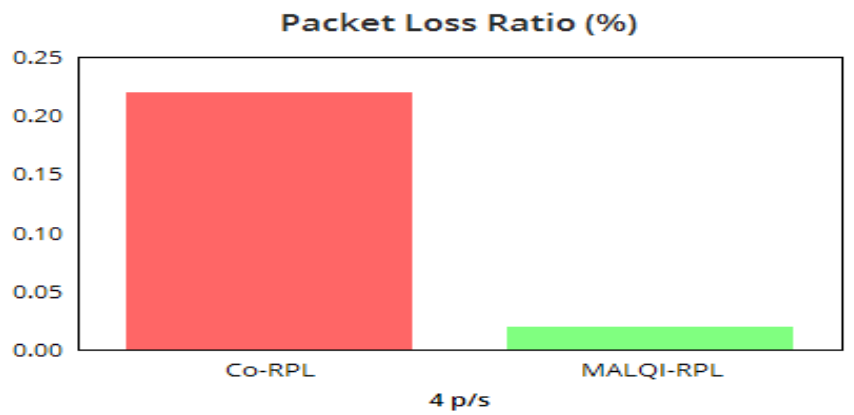

Fig. 12. Packet Loss ratio comparison between MALQI-RPL and Co-RPL with $4 \mathrm{p} / \mathrm{s}$ data rate

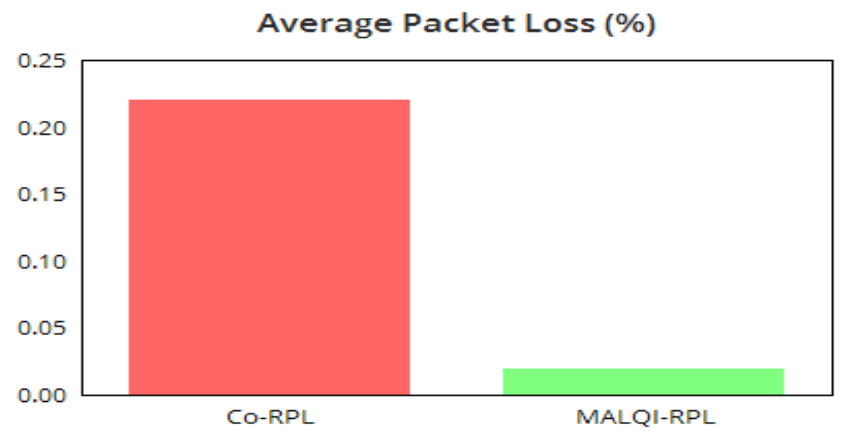

Fig. 13. Average packet loss ratio comparison between MALQI-RPL and Co-RPL

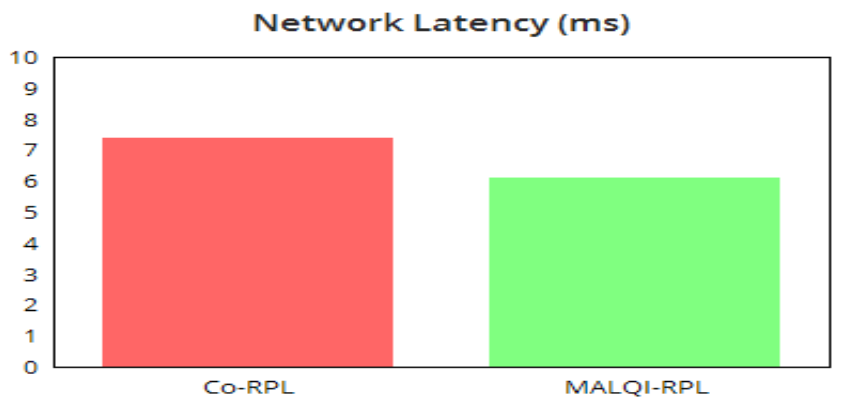

Fig. 14. Network latency comparison between MALQI-RPL and Co-RPL

Fig. 14 presents the comparison of the network latency performed by both our proposed MALQIRPL and Co-RPL routing protocol. Here, it can be found that our proposed routing protocol performs better than the existing Co-RPL routing protocol. Thus, observing overall results and their respective significances, it can be found that the proposed routing protocol (i.e., MALQIRPL) can be efficient to enable delay resilient, reliable and QoS oriented communication over LLNs under mobile node conditions or mobile network topology. The overall conclusion of the presented research is given in the next section.

\section{Conclusion And Future Scope}

In recent years the global human society has witnessed the exponential rising demands for quality of service (QoS) and Quality of Experience (QoE) oriented wireless communication systems. Being decentralized and infrastructure less in nature Wireless Sensor Networks (WSNs) has established itself as one of the potential solution for different communication purposes. The 
recently developed routing protocol for low power lossy networks (LLNs), commonly known as RPL has performed better, especially for IoT ecosystems. However, the unavailability of mobility in native RPL routing protocol limits its applicability. The predominant reason behind it is the mobility caused dynamism that introduces topological changes, network parameter variations and thus makes network management highly intricate. Enabling best parent selection and forwarding path formation in mobile-RPL can be of paramount significance to enable an array of communication systems. With this motivation, a novel and robust Multi-Constraints Adaptive Link Quality Index (MALQI) based parent node and forwarding path selection model is developed for mobile-RPL based LLNs. In this paper, two significant network parameters dynamic RSSI and ETX have been used to decide best parent node from the proactively managed parent-set node table. In this approach, the developed MALQI based mobile-RPL operates in parallel to the link layer and once detecting link-outage it executes node discovery using proposed MALQI model. In the proposed model, each RPL node maintains data backup, and once detecting any link-outage and forming alternate best forwarding path, it transmits data through that path, and thus avoids performance degradation. Additionally, to avoid any control message caused overheads and resource (time, memory, energy) consumption, the trickle timer has been disabled and fixed at the interval of $2 \mathrm{~ms}$. It has significantly improved the computational efficiency of the proposed routing protocol. The use of ETX that is having direct relation between RSSI and associated packet delivery ratio (PDR) as the criteria for the best parent node and path selection strengthens proposed routing protocol to assure reliable and fault resilient transmission over mobile-RPL. Additionally, the consideration of RSSI adaptive mobile node positioning and interanchor-mobile node communication enables the proposed system reliable and further enables fault-tolerant communication over RPL. The simulation results obtained confirm that the proposed MALQI-RPL routing protocol exhibits an improvement of 5\% better packet delivery ratio/received ratio, around 1\% low latency and around 5\% low packet drop compared to standard RPL even under varying packet rate or payload conditions. It exhibits its robustness for realworld burst type communication. The proposed routing model has performed better than not only the standard RPL based routing, but also recently proposed state-of-art technique called Co-RPL for mobility based RPL. Thus, the overall results confirm that the proposed routing model exhibits higher PDR, low loss and low latency even with different payload condition and dynamic network statistics. In the future, other objective functions can also be explored or derived to achieve performance with real time test-bed applications.

\section{REFERENCES}

[1] Heinzelman W., Kulik, J., Balakrishnan, H.: Adaptive Protocols for Information Dissemination in Wireless Sensor Networks. In proc. of the 5th ACM/IEEE Mobicom Conference (MobiCom '99), Seattle, WA, August, pp. 174-85 (1999)

[2] Intanagonwiwat, C., Goninan R., Estrin, D.: Directed diffusion: a scalable and robust communication paradigm for sensor networks. In proc. of the ACM MobiCom '00, Boston, MA, pp. 56-67. Y (2000)

[3] Yao, Y., Gehrke, J.: The cougar approach to in-network query processing in sensor networks. In ACM SIGMOD Record, vol. 31, no. 3. (2002)

[4] Heinzelman, W., Chandrakasan A., Balakrishnan, H.: Energy-Efficient Communication Protocol for Wireless Microsensor Networks. In proc. of the 33rd Hawaii International Conference on System Sciences (HICSS '00) (2000)

[5] Lindsey, S., Raghavendra, C., PEGASIS: Power-Efficient Gathering in Sensor Information Systems. In proc. of the IEEE Aerospace Conference. vol. 3, pp. 1125-1130. (2002) 
International Journal of Computer Networks \& Communications (IJCNC) Vol.10, No.5, September 2018

[6] Winter, T., Thubert, P., Brandt, A., Clausen, T., Hui, J., Kelsey, R., Levis, P., Pister, K., Struik R., and Vasseur, J.: RPL: IPv6 Routing Protocol for Low power and Lossy Networks. RFC 6550, IETF ROLL WG. (2012)

[7] Vasseur, J.P: Terminology in Low power And Lossy Networks. Draftietf-roll-terminology06.txt. (2011)

[8] Lee, K.C., Sudhaakar, R., Ning, J., Dai, L., Addepalli, S., Vasseur, J. P., Gerla, M.: A Comprehensive Evaluation of RPL under Mobility. Hindawi Publishing Corporation International Journal of Vehicular Technology, Vol. 2012.

[9] Vasseur, J., Dunkels, A.: Interconnecting Smart Objects with IP: The Next Internet. Morgan Kaufmann, 1 edition. (2010)

[10] Tsiftes, N., Eriksson, J., Dunkels, A.: Low-power wireless ipv6 routing with contikiRPL. in Proceedings of the 9th ACM/IEEE International Conference on Information Processing in Sensor Networks, ser. IPSN '10. New York, NY, USA: ACM, pp. 406- 407. (2010)

[11] Pavkovi'c, B., Theoleyre, F., Duda, A.: Multipath opportunistic RPL routing over IEEE 802.15.4. in Proceedings of the 14th ACM International Conference on Modeling, Analysis and Simulation of Wireless and Mobile Systems, ser. MSWiM '11. New York, NY, USA: ACM, pp. 179-186. (2011)

[12] Duquennoy, S., Landsiedel, O., Voigt, T.: Let the tree bloom: Scalable opportunistic routing with ORPL. in Proceedings of the 11th ACM Conference on Embedded Networked Sensor Systems, ser. SenSys '13. New York, NY, USA: ACM, pp. 2:1-2:14. (2013)

[13] Hong, K.S., Choi, L.: Dag-based multipath routing for mobile sensor networks. in ICT Convergence (ICTC), 2011 International Conference on, pp. 261-266. (2011)

[14] Safdar, V., Bashir, F., Hamid, Z., Afzal, H., Pyun, J.Y.: A hybrid routing protocol for wireless sensor networks with mobile sinks. In Wireless and Pervasive Computing (ISWPC), 2012 7th International Symposium on, pp. 1-5. (2012)

[15] Ben Saad, L., Chauvenet, C., Tourancheau, B.: Simulation of the RPL Routing Protocol for IPv6 Sensor Networks: two cases studies. in International Conference on Sensor Technologies and Applications SENSORCOMM 2011. Nice, France: IARIA (2011)

[16] Ben Saad, L., Tourancheau, B.: Sinks Mobility Strategy in IPv6- based WSNs for Network Lifetime Improvement. in International Conference on New Technologies, Mobility and Security (NTMS). Paris, France: IFIP, pp. 1-5. (2011)

[17] Korbi, I.E., Ben Brahim, M., Adjih C., Saidane, L.A.: Mobility Enhanced RPL for Wireless Sensor Networks. 2012 Third International Conference on the Network of the Future (NOF), Gammarth, pp. 1-8. (2012)

[18] Tripathi, J., de Oliveira, J., Vasseur, J.: A Perfor- mance Evaluation Study of RPL: Routing Protocol for Low Power and Lossy Networks. Information Sciences and Systems (CISS), 2010 44th Annual Conference on, pp. 1-6. (2010)

[19] Tsiftes, N., Eriksson, J., Dunkels, A.: Poster Abstract: Low-Power Wireless IPv6 Routing with ContikiRPL. IPSN10, Stockholm, Sweden. p. 1216. (2010)

[20] Kharrufa, H., Al-Kashoash, H., Al-Nidawi, Y., Mosquera, M.Q., Kemp, A.H.: Dynamic RPL for multi-hop routing in IoT applications. 2017 13th Annual Conference on Wireless On-demand Network Systems and Services (WONS), Jackson, WY, USA, pp. 100-103. (2017)

[21] Gaddour, O., Koubäa, A., Rangarajan, R., Cheikhrouhou, O., Tovar, E., Abid, M.: Co-RPL: RPL routing for mobile low power wireless sensor networks using Corona mechanism. Proceedings of the 
International Journal of Computer Networks \& Communications (IJCNC) Vol.10, No.5, September 2018

9th IEEE International Symposium on Industrial Embedded Systems (SIES 2014). Pisa. pp. 200-209. (2014)

[22] Somaa, F., Korbi, I. E. 1., Adjih, C., Saidane, L.A.: A modified RPL for Wireless Sensor Networks with Bayesian inference mobility prediction. 2016 International Wireless Communications and Mobile Computing Conference (IWCMC). Paphos, pp. 690-695. (2016)

[23] Barcelo, M., Correa, A., Vicario, J. L., Morell, A., Vilajosana, X.: Addressing Mobility in RPL With Position Assisted Metrics. In IEEE Sensors Journal, vol. 16, no. 7, pp. 2151-2161. (2016)

[24] Lohith, Y.S., Narasimman, T.S., Anand, S.V.R., Hedge, M.: Link Peek: A Link Outage Resilient IP Packet Forwarding Mechanism for 6LoWPAN/RPL Based Low-Power and Lossy Networks (LLNs). IEEE International Conference on Mobile Services. New York. NY. pp. 65-72. (2015)

[25] Lee et al., K.C.: RPL under Mobility. In proc. of the IEEE Consumer Communications and Networking Conference (CCNC) (2012)

[26] Levis, P., Clausen, T., Hui, J., Gnawali, O., Ko, J.: The Trickle Algorithm. RFC 6206 (Proposed Standard). (2011)

[27] Gungor, V.C., Hancke, G.P.: Industrial Wireless Sensor Networks: Applications, Protocols, and Standards. CRC Press, Inc., Boca Raton, FL, USA, 1st edition. (2013)

[28] Raman, B., Chebrolu, K., Madabhushi, N., Gokhale, D.Y., Valiveti, P. K., Jain, D.: Implications of link range and (in) stability on sensor network architecture. WiNTECH '06, ACM. New York, NY. USA. pp. 65-72. (2006)

[29] Srinivasan, K., Levis, P.: RSSI is under appreciated. In Proceedings of the Third Workshop on Embedded Networked Sensors. EmNets. (2006)

[30] Sneha K, Dr. B.G.Prasad.: An Efficient Hand-off Optimization Based RPL Routing Protocol for Optimal Route Selection in Mobility Enabled LLNs in IEEE International Conference on Global Trends in Signal Processing, Information Computing and Communication (ICSPICC-2016), Jalgaon, India: IEEE. (2016). 